ARTICULOS

ORIGINALES

\title{
Hemorragia suprarrenal en el recién nacido
}

DRES. SAMUEL BENVENISTE * **, JORGE LAS HERAS*, DAVID MIRK1N *, ADRIANA FUENTES ***.

livt Roduccióx. La hemorragia suprarrenal en ef reción nacido, aun siendo causa poco frecuente de muerte, constituye un haliazgo de cierta cuantía asociado a condiciones patológicas del neonato.

Los factores etiopatogénicos tienden a explicarse en parte por las características anatómicas de la glándula en el recién nacido (16-17) y por otro lado, en relación con alteraciones producidas por la infección y la anoxia. (9-10.14). Por to común la casuístić existente se refiere a hallazgos de hemorragias unilaterales severas, (5-8-1522) las cuales por sí mismas constituyen una entidad patológica.

Nuestro interés fue el de revisar todos los casos de muertes neonatales en los que se descubrió durante el estudio anatomopatológico algún grado importante de hemorragia suprarrenal.

Material y método. Se analizaron retrospectivamente 352 autopsiats de recién nucidos realizadas durante un periodo de 5 años (1969-1973). Se encontraron 21 de ellos con hemorragia suprarrenal asociada a otras patologías, lo cual representa un $5,96 \%$ del total de las muertes investigadas.

En cada niño con hemorragia suprarrenal, se analizaron los antecedentes clínicos, patología asociada, estudio bacteriológico, tratamiento recibido, descripción macroscópica y estudio microscópico anatomopatológico, con lás técnicas de rutina y coloraciones especíticas para fibrina.

Resultados. Del grupo estudiado, 7 eran rcciền nacidos pretérmino y los $\mathrm{I} 4$ restantes recién nacidos de término.

\footnotetext{
Servicio de Anadosnia Patológica, Huspilal Roberto del Rio. Santiago de chile.

s:- Trabajo de prósentálión a Ia Sociedad Chilena de Pediatría.

"T** Ténologo Móxtico, Hospjial Ruberto del Rís, Santiago de Chitl:
}

La edad de muerte varió entre la una hora y los 22 días de vida, con un promedio de 4,6 días, siendo el $85 \%$ menor de 10 días. Los datos clínicos se evaluaron de acuerdo a la enfermedad fundamental y sólo en dos casos se planteó clínicamente la posibilidad de hemorragia suprarrenal, los que por su interés se describen a continuación:

Primer caso. Recién nacido, con septicemia desde el $2^{\circ}$ día de vida que evolucionó bien hasta el $8^{\circ}$ día en que bruscamente presentó palidez, dificultad respiratoria, crisis de apnea y falleció. En la autopsia, además de la hemorragia suprarrenal masiva, se observó trombosis de las venas renales.

Seziundo caso. Recién nacido que desde las 11 horas de vida presentó dificultad respiratoria progresiva, habiéndose palpado dos masas lumbares, la matyor a izquierda. Falleció a las 24 horas de vida y en la autopsia se encontró hemorragia suprarrenal bilateral, hemorragia pulmonar y hemotórix.

En los 21 casos estudiados se encontró la siguiente patología: (cuadro 1).

$$
\text { CUADRO } 1
$$

HEMORRAGIA SUPRARRENAL EN EL RECIEN NACIDO. PATOLOGIA ASOCIADA

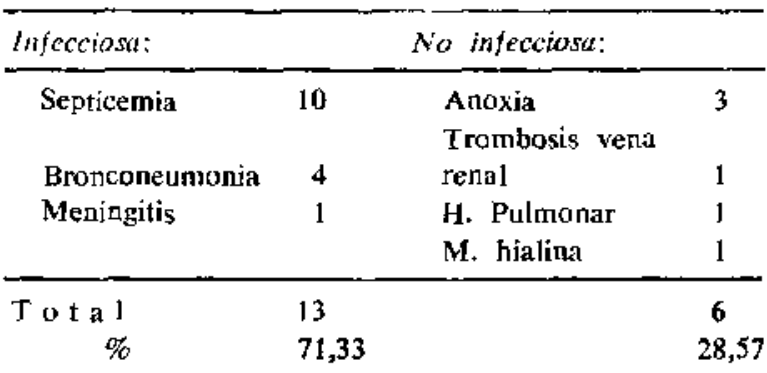


C U A D O 2

HEMORRACIA SUPRARRENAL EN EL RECIEN NACIDO. GRADOS DE HEMORRAGIA

\begin{tabular}{ccccc}
\hline $\begin{array}{c}\text { Biloteral } \\
\text { masiva }\end{array}$ & $\begin{array}{c}\text { Bilateral } \\
\text { focal }\end{array}$ & $\begin{array}{c}\text { Unilateral masiva } \\
\text { Derecha Izquierda }\end{array}$ & Total \\
\hline 11 & 9 & 0 & 1 & 21 \\
\hline
\end{tabular}

En el estudio bacteriológico de los niños, en tres se obtuvo germen patógeno (Acromobacter, Escherichia coli y Piociánico, respectivamente).

Los niños con infecciones recibieron tratamiento antibiótico y en ninguno de los 21 casos se administró terapia esteroidal. El estudio anatomopatológico de las suprarrenales evidenció distintos grados de hemorragia (cuadro 2 ).

Los cortes histológicos además de la hemorragia mostraron: trombosis sinusoidal en 7 casos, necrosis de la zona en 7 casos, degeneración quística de la corteza en 1 caso, y calcificación en 1 calso.

En todos ellos la porción yuxtamedular de la corteza fetal fue la zona más comprometida, extendiéndose la hemorragia cn casi todos al resto de la corteza fetal y medular.

La corteza adulta fue por lo general la zona más conservada.

Comentario. Las características clínicas de la hemorragia parecen ser muy variables y dependen en alguna medida de la extensión de la lesión y el grado de compromiso de la glándula. En nuestros casos no siempre los datos clínicos fueron bien precisados, no pudiendo obtenerse en muchos de cllos una adecuada correlación clínicopatológicá.

La mayoria de los autores señalan que no hay signos diagnósticos de la hemorragia suprarrenal. Sólo cuando la hemorragia es muy extensa, puede dar signos de colapso con distensión abdominal (4-5). En estos casos debería hacersc cl diagnóstico diferencial con otras causas de hemorragia intraabdominal en el recién nacido (hepática, esplénica y renal) (1-2-6-7).

En otras ocasiones, la palpación de una masa en algunos de los flancos, plantea el diagnóstico diferencial con tumor de Wilms, neuroblastoma, o malformación renal (1).

El análisis de los antecedentes obstétricos, un examen físico cuidadoso y el registro adecuado de los hallazgos clínicos podrían permitir la sospecha de este cuadro. La realización de cstudios radiológicos (pielogralía de excreción y/o retroneumoperitoneo), pueden facilitar el diagnóstico (2-822).

Analizando la patología asociada se observa que el mayor número de casos $(71,33 \%)$ se acompañó de infecciones graves. En el grupo sin infección prevaleció la anoxia perinatal. Ejl estudio anatonopatológico de las glándulas suprarrenales mostró hemorragia de distintos grados. La mayoría tenía hemorragia bilateral masiva y solamente uno de ellos presentaba hemorragia unilateral.

Algunos autores (9-10) para explicar la hemorragia, invocan el fenómeno de Shwartzman asociado a la trombosis de los sinusoides en los casos de infección severa, siendo esto aún más factible cuando se asocia a coagulación intravascular diseminada. En 7 dc nuestros casos se cvidenció la ptesencia de trombos de fibrina en capilares venosos (Fig, 1, 2, 3).

También se puede pensar que lá localización del trombo es las suprarrenales hiperémicas de niños con septicenia sea un accidente del stress en respuesta a la infección (9).

Por otra parte, algunos autores descartan la posibilidad de la trombosis arterial como causa

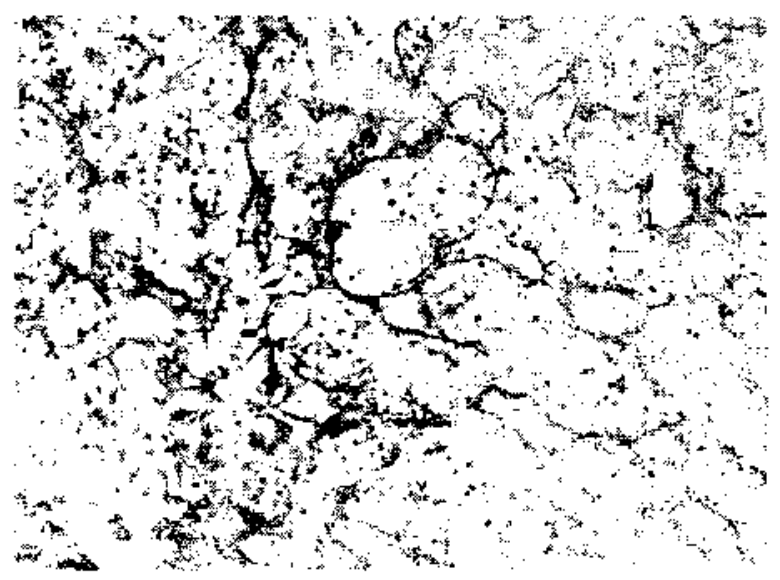

Mallas de librina que rocicar trabículas supriartenalcs con necrosis extensa. $25 \mathrm{x}$. Hemaloxilina fosíotúngsliza.

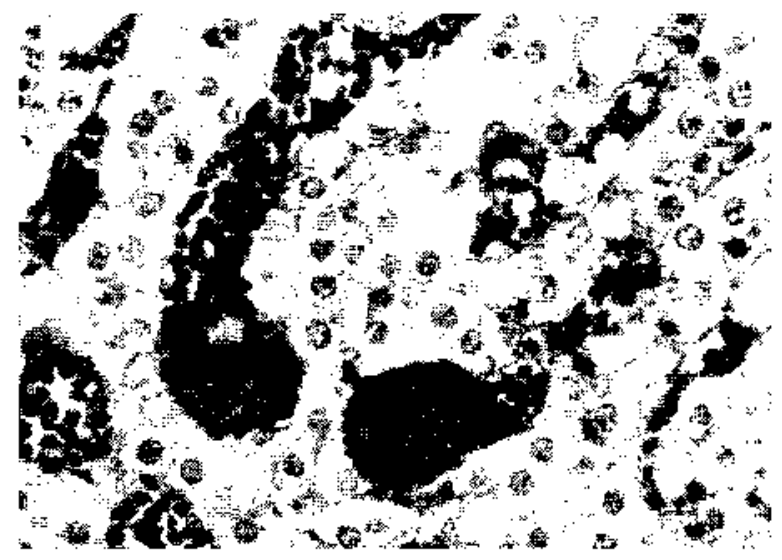

Capjatres congestivos y comienzes de formaxion de trombos. HWC x. Hematoxílira lossotúngstica. 


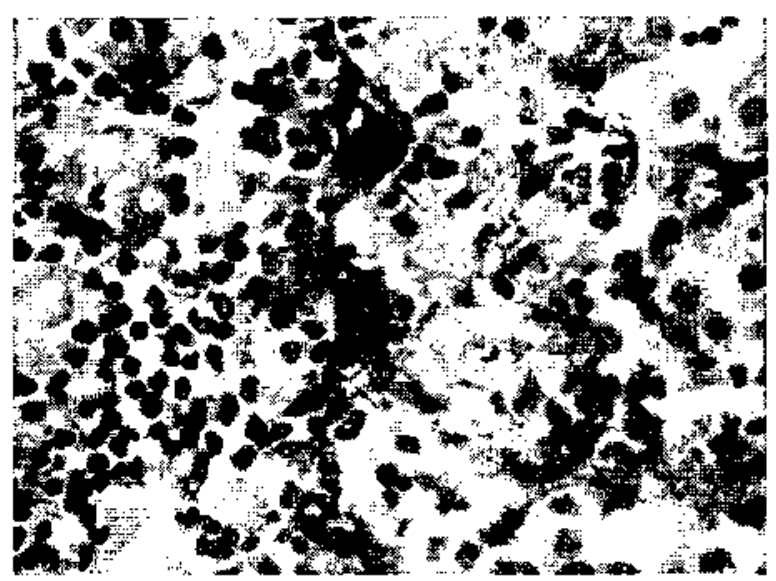

Trombos de fibtina $y$ hontoragia inicial ([lechas). 400 x, FlemaLoxílina fosfotúngstica

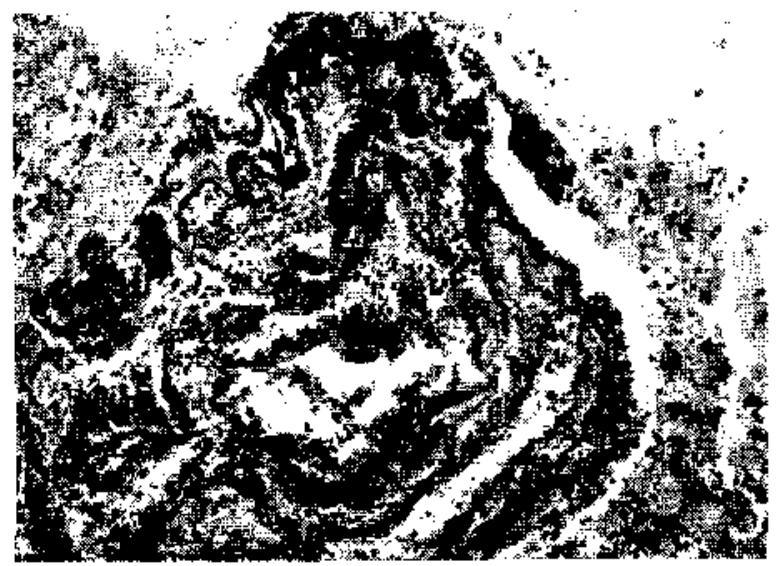

Formación de capas concéntricas de fibrina en trumbo que ocupa la vena central de la medular suprarrenal. 25 x. HemaIoxilina lusfutúngestica.

(11), sobre todo considerando que la glándula suprarrenal recibc irrigación arterial numerosa, a partir de tres vasos principales y otros más pequeños (12). Sería más adecuado pensis en la posibilidad de obstrucción venosa, ya quc se ha visto, al igual que en la trombosis de la vena renal, que la obstrucción en el drenaje venoso (Fig. 4) produce distensión progresiva de la glándula y hemorragia, con posterior destrucción de tejido suprarrenal (13).

Boyd, en 1967 (14) describió la existencia de lesiones tromboembólicas diseminadas en niños nacidos muertos y en otios fallecidos dentro de las primeras 48 horas de vida. Estas lesiones afectaban sobre todo las stiprarrenales del segundo grupo y consistían en masas de fibrina que comprometían los capilares corticales y los senos medulares.

Fox en 1969 (11-15) en relación con lo plantcado por Boyd, describió como probable la asociación entre trombosis del sistema venoso de la cápsula $\mathrm{y}$ hemorragia supratrenal masiva.
Otros autores (16-17) han tratado de demostrar que las características anatómicas de las suprarrenales del recién nacido predisponen a la hemorragia, a través de mecanismos vasculares reJacionados con la falta de desarrollo de la glándula.

La mayor incidencia de hemorragia masiva de la glándula suprarrena! derecha ha sido explicada por las características de su drenaje venoso (18).

Se ha visto que la vena correspondiente desemboca directamente en la vena cava inferior, to que sometería a la glándula a cambios de presiones mayores y provocaría distensión pasiva de la misma y hemorragia. En nuestra revisión, el único caso de hemorragia unilateral comprometió li glándula del lado izquierdo.

La asociación en 5 casos de enterocolitis necrosante y hemorragia suprarrenal (35\% de los niños con infección) es sugerente de alguna callsa etiopatogénica común. Ambos podrían estar en refación con el fenómeno de Shwartzman $(9)$, con cl stress y tambiér con la hipoxia.

Esta última participaría en la producción de hemorragia al provocar lesión endotelial y des. encadenar secundariamente el mecanismo de cosgulación.

Se debe destacar que el hallazgo postmortem de hemorragia suprarrenal no constituye sino una parte del total de dicha patología en el recién nacido. Esto hace difícil precisar con exactitud qué porcentaje, en recién nacidos con patología infecciosa grave o anóxica. desarrollan dentro de su evolución algún tipo de hemorragia supparrenal.

No es infrecuente descubrir calcificaciones sulprarrenales en radiografías de abdomen (19), tomadas por otras causas durante la infancia, en que existió hemorragia de liz glándula y esta no tue reabsorbida precozmente. Esto permite especular sobrc la posible asociación entre hemorragia suprarrenal y algunos tipos de insuficiencia de Ia glándula que se manifiestan tardíamente. Se describen algunos casos de niños que presentaron cuadros addisomianos en relación con calcificaciones de suprarrenales, (19-21), atribuible a hemorragia y en los que no se demostró antecedentes de tuberculosis o tumor.

Es probable que la existencia de un grado importante de hemorragia suprarrenal signifique un grado variable de insuficiencia de la glándula, aunque no sicmpre se encuentren con posterioriridad evidencias clínicas de laboratorio.

\section{RESUMEN}

Se analizaron retrospectivamente 352 autopsias de recién nacidos encontrándose 21 casos $(5,96 \%)$ con henorragia suprarrenal asociada a otras patologias. 
Se destaca la asociación con infecciones severas, $y / 0$ anoxia. Se discute la etiopatogenia $y$ en especial la participación de la trombosis venosa en la produccion de hemorragia.

Conviene destacar la importancia del diagnóstico precoz y la existencia de elementos clínicos $y$ radiológicos que 6 permiten.

\section{SUMMARY}

352 autopsis of newborns were analized in retrospect finding 21 cases $(5.96 \%$ ) with idtenal haemorrhage associnted to other pathology.

The associtition of adrenal haemorrhage with severe infections and anoxia i; remarked.

The ethiology of this condition is discussed, particulary the participation of venous thrombosis in the generation of the haemorrhage.

The importance of the carly diagnosis and the clinical and radiologycal elements that would make it possible are stressed.

\section{BibLIOGRAFÍA}

1.-Gwin J. L., Burness G. R. Massive adronal haemorrhuge in the newborn. Amer. J. Dis. Child. 109: $259,1965$.

2.- Siegel B. S. Adrenal haemorrhage in newborn. JAMA, 177: 273, 1961.

3.- Schaffer A. J., Alery M. E. Diseases of the Newborn, 435: 439, Third Edition, Saunders Co., 1971.

4.- Snelling C. E., and Erb, L. H. Haemorrhage and Subsequent cakification of Suprarrenal. J. Pediat., 6: $22,1935$.

5.-Bianchedi $S$. and Soane F. Massive haemorrhage into right adrenal cortex in neonates, simulating abdominal tumour. Operation cure, Miverva Pediat. 14: 1285,1962 .

6.- Grunewalk, $P$. Rupture of Liver and Spleen in newborn Infant, J. Pediat. 33: 195, 1948.

7.-Lundguisf, B. Infrathoracic und Intra-abdominal Hiternorhage in the newborn. Acta Obstet. Gynec. Scand. 9: 331, 1930.

8.-Gelnn 3. F. Neonatal adrenal haemorrhage, J, Urol. (Baltimore) 87: 639, 1962.
9.-Margarcten W. Nakat. H, and Landing B. Septicemic Adrenal Huemorrhage, Amer. J. Dis. Child. 105: $346,1963$.

10.-May C. D. Circulatory Failure (Shock) in Fulminant Meningococcal Infection, Pediatrics 25: 316 , 1960.

11.-Fox. B. Unilateral adrenal necrosis and hasmorrhage following operations on the stomach, Journal of Pathology, 97; 127, 1969.

12.-Gagnon, $R$. The arterial supply of the human adrenial glond Révue Canadienne de Biologie, 16: 421, 1957.

13.- Black, I. and Williams $D$. Natural history of adre. nat haterrorhage in the newborn. Archives of Dissease in Childhood, 48; 183, 1973.

14.-Boyd. J. F. Disseminated fibrin thromboembolism among neovates dying within 48 hours of birth, Archives of Disease in Childhood, 42: 401, 1967.

15.-For B. Adrenal hacmorrhage and necrosis resulting from abdominal operations, Lancet 1: 600, 1969.

16.-Dobbie, J, W, and Symingron, $T$. The human adrenal gland with special reference to the vasculature, Journal of Endocrinology, 34: 479, 1966.

17.-- Meinivara, $O$. On the strucule of the human suprarenal vein. Annales Medicinae Internae Fenniae, 43: suppl. 19, 1954.

18. - Gagnon $R$. The venour drainage of the human adrenal gland. Révue Cantdiende de Biologic, 14: 350, 1955-56.

19.- Keuth $U$. and The'se E. Spontaneus hypoplycemia and salcificution of the adrenals in young children Z. Kinderheilkd 100: 113, 1967.

20.-CGross $M$, et al Diagnosis and treatment of neoratal adrenal haemorrbage. J. Pedjat Surg. 2; 308, 1967.

21.-Wogner A. C. Bilateral haemorrhage pseudocyst of the adrenal glands in a newborn. Amer. J, Ruentgenol. 86: 540, 1961.

22.-Brown, B. St. J. at al. The radiologic features of acute massive adrenal haemorrhage of the newborn. J. Canad. Agg. Radiol. 13: 100, 1962. 